\title{
Danser avec le sexe. Érotisme et exotisme dans la réception parisienne du tango (1907-1914)
}

Dancing with le sexe: eroticism and exoticism in the Parisian experience of tango (1907-1914)

\section{Rafael Mandressi}

\section{(2) OpenEdition}

Journals

\section{Édition électronique}

URL : https://journals.openedition.org/clio/13713

DOI : 10.4000/clio.13713

ISSN : $1777-5299$

\section{Éditeur}

Belin

Édition imprimée

Date de publication : 1 décembre 2017

Pagination : 87-110

ISBN : 978-2-410-00859-3

ISSN : $1252-7017$

\section{Référence électronique}

Rafael Mandressi, «Danser avec le sexe. Érotisme et exotisme dans la réception parisienne du tango (1907-1914) », Clio. Femmes, Genre, Histoire [En ligne], 46 | 2017, mis en ligne le 01 décembre 2020, consulté le 29 avril 2022. URL : http://journals.openedition.org/clio/13713 ; DOI : https://doi.org/ 10.4000/clio.13713 


\title{
Danser avec le sexe. Érotisme et exotisme dans la réception parisienne du tango (1907-1914)
}

\author{
Rafael MANDRESSI
}

«Pour danser ça, ne faut-il pas être couché ?» Voilà, d'après Gabriel-Louis Pringué (1885-1965), chroniqueur de la haute société parisienne de la première moitié $\mathrm{du} \mathrm{XX}^{\mathrm{e}}$ siècle, ce que la comtesse Mélanie de Portalès aurait murmuré à l'oreille de «l'académicien distingué » qui se trouvait à côté d'elle, alors que devant eux un couple s'essayait au tango ${ }^{1}$.

L'anecdote n'est peut-être pas véridique, mais elle traduit en tout cas un des aspects qui ont marqué la première réception européenne du tango, dans les années immédiatement antérieures à la Première Guerre mondiale : le caractère fortement sexuel associé à cette danse étrangère. L'épisode raconté par Pringué témoigne aussi du lieu, à la fois géographique et social, où le tango fut d'abord et principalement accueilli: Paris, ses milieux aristocratiques et grand-bourgeois, qui furent le foyer d'irradiation de la découverte de la «nouvelle danse » et de l'engouement qu'elle suscita dans d'autres grandes villes, en Europe et aux États-Unis ${ }^{2}$. Paris fut en effet le centre du premier cycle de diffusion internationale du tango et, à ce titre, une étape fondamentale dans l'établissement de représentations, mais aussi de pratiques, qui en ont longuement façonné l'image publique. On peut même aller au-delà, et constater les effets significatifs et durables de ce premier moment de l'histoire parisienne du tango sur le plan chorégraphique, ainsi que dans

1 Pringué $1948: 47$.

2 Humbert 1995 sur l'arrivée du tango à Paris jusqu'en 1920; Buch 2017 sur la condamnation du tango par l'Église de France en janvier 1914. 
la très vaste littérature que constituent, au premier chef, les dizaines de milliers de paroles du tango-chanson ${ }^{3}$.

L'objet de cet article est plus modeste et plus restreint que cela. Il s'agit de cerner, à travers des discours dont on dira le caractère socialement situé, la construction de la nature érotique du tango, en parallèle avec l'enjeu de l'exotisme - obscurité étrangère des origines d'une danse dont on s'approprie, dans un rapport ambivalent avec la distance, aussi bien géographique que sociale, qu'elle est censée introduire et exprimer. Le tango, avatar de la sexualisation de l'altérité qu'on incorpore en l'apprivoisant, est, comme d'autres danses l'ont été à d'autres moments, un foyer d'expression, mais aussi, le cas échéant, de réaménagements ou renégociations des rapports de genre. Sophie Jacotot l'a montré, pour le cas de Paris, au sujet des danses de société des Amériques de l'entre-deux-guerres - dont le tango 4 . Une des spécificités de la période précédente, dans les années 1911-1914, est la nette domination du tango dans le répertoire des danses " exotiques », ainsi que son fort ancrage dans les milieux mondains, qu'il convient d'ailleurs de saisir également dans leur économie de genre: «Un salon, c'est d'abord une femme», écrit Anne MartinFugier, en mettant en avant un des aspects de la féminisation de la mondanité sous la III République, qui sera aussi l'écosystème d'accueil du tango au début du XX⿳亠丷厂 siècle $^{5}$.

Une partie des sources pour une étude qu'on ne fait ici qu'engager, le dit par sa nature même : la presse "féminine » est un des lieux privilégiés de production de discours, très souvent normatifs, autour de la danse du tango, ses connotations, les modalités et les espaces de sa consommation, les enjeux de son acclimatation, son rôle éventuel dans l'altération des repères de la bienséance. À cette presse s'ajoute une production imprimée qui, d'une part, en prend en quelque sorte le relais - chroniques et mémoires d'acteurs et/ou observateurs de la vie mondaine -, et qui, d'autre part, rend compte de l'entrée du tango dans le répertoire des

3 Vilariño (poétesse et critique littéraire uruguayenne) 1964, 1965 et 1981.

4 Jacotot 2007, 2008 et 2013 : 331-385; Décoret-Ahiha 2004: 63-10 et 2005; Suquet 2012 ; Savigliano 1995.

5 Martin-Fugier 2003 : 8 ; Mension-Rigau 1994 ; Bravard 2013. 
danses admises au sein de la société des salons - les manuels de danse, notamment. Des publicités, des textes accompagnant les partitions publiées, des romans, des pièces de théâtre, un discours académique $^{6}$, les paroles des rares tangos écrits à Paris à cette époque, font également partie du répertoire de sources textuelles à exploiter, que complètent quelques films et, surtout, une abondante documentation iconographique - photographies, dessins de presse, cartes postales, caricatures ${ }^{7}$. Il existe aussi toute une littérature produite a posteriori, dans les années 1920 ou 1930, voire plus tard - l'ouvrage de Pringué dont on a tiré la citation qui ouvre notre propos, par exemple -, qui revisite, sous la forme de mémoires de témoins, d'observateurs ou de protagonistes, la «vie parisienne» et, pour ce qui nous intéresse ici, son premier « moment tango $»^{8}$.

\section{Les premiers pas}

Les difficultés d'une approche historique des premières étapes du tango sont celles rencontrées pour toute pratique élaborée dans l'anonymat des marges. Au moins jusqu'au début du XX ${ }^{\mathrm{e}}$ siècle, le tango est en effet une affaire marginale dans un double sens : les marges de la société et les marges des villes, Montevideo et Buenos Aires ${ }^{9}$. Une obscurité relative entoure les «origines» du tango en tant qu'espèce chorégraphique et musicale; son arrivée en Europe est tout autant marquée par l'incertitude. Une version aussi tenace qu'improbable voudrait que des partitions du tango La Morocha, du pianiste et violoniste uruguayen Enrique Saborido (1878-1941), aient été introduites à Marseille lors d'une escale de la frégate "Presidente Sarmiento », navire-école de la marine de guerre argentine, en $1906^{10}$.

6 Richepin 1913 ; Gasió 1999 sur Richepin et le tango.

7 Caricatures de Sem Tangoville sur mer (1913b), 24 planches couleur ; Tango-Rausch 1913 in Lustige Blätter de Berlin, dix planches in-4.

8 Georges-Michel 1920 ; Mourgue 1920 ; Warnod 1922 ; Germain 1923 ; Gramont 1923 et 1966 ; Cocteau 1935 ; Fouquières 1951 et 1953-1959.

$9 \quad$ Héctor \& Luis Bates 1936 ; Vega 1936, 1952, 1967 et Ayestarán 1953 ; Antología del tango rioplatense 1980 ; Goldman 2008 ; Castro 1990 ; Chasteen 2004.

10 Entretien avec Saborido. Sur les voyages de la frégate «Sarmiento », Los Viajes de la «Sarmiento », 1931. 
Le pianiste et compositeur argentin Alberto López Buchardo (18821948) ne saurait davantage être considéré, par son premier séjour parisien en 1903 ou 1905, comme l'introducteur du tango dans la capitale française - il en fut plus tard un des promoteurs -, et tout semble indiquer que la date à retenir est celle de 1907, lorsque la firme Gath \& Chaves de Buenos Aires envoie à Paris, afin d'enregistrer des phonogrammes, le violoniste uruguayen Alfredo Gobbi (1877-1938) et son épouse la chanteuse chilienne Flora Rodríguez (1883-1952). Les Gobbi enregistrent entre autres des tangos, ce qui n'avait en soi aucune signification dans le contexte parisien - la firme Gath \& Chaves ne visait pas ce marché mais bien ceux de Buenos Aires et Montevideo ; or le couple restera dans la capitale française pendant sept ans et, dès 1909, un tango composé par Gobbi, El mamao, est enregistré par la Bande Pathé. En 1908, l'Orchestre Tzigane du restaurant du Rat mort, situé sur la place Pigalle, avait enregistré El choclo, d'Angel Villoldo (1861-1919), lui aussi envoyé à Paris par la firme Gath \& Chaves. En 1907 déjà, un orchestre dirigé par Auguste Bosc († 1945) avait pourtant enregistré le tango Joaquina, du pianiste Juan Bergamino (1875-1959) ${ }^{11}$.

En 1909, Eugène Giraudet, maître de danse, chorégraphe, musicien et auteur, entre autres, d'un Traité de la danse en 1890, fait allusion au tango dans le Journal de la danse et du bon ton, qu'il a fondé en 1905, en le définissant comme une "sorte de danse américaine tout nouvellement introduite en France », dont le «mouvement est à 2/4 », qui « se divise en deux parties, dont l'une est marchée et l'autre est valsée »12. En 1910, Mistinguett (Jeanne Florentine Bourgeois, 1875-1956) aurait dansé un tango dans un spectacle de music-hall avec Barthélemy Bottallo, directeur de l'Académie de danse de la Sorbonne, et auteur en 1912 d'un Guide du bon danseur où le tango figure parmi les danses expliquées ${ }^{13}$. En 1911, Bernabé Simarra, qui s'était taillé une belle réputation en remportant plusieurs concours de

11 Alain Boulanger (dir.), Tango : le tango à Paris 1907-1941, 1994, Label Frémeaux et associés, 2 CD plus livret.

12 Giraudet 1909 : 2413 et Eugène Giraudet, «Le Tango tel qu'on le danse dans les salons et familles ", Journal de la danse et du bon ton, 1914, n²41 à n²50: 41164133 ; Alfredo Gobbi, Qué hacés, pulenta, El sanducero, Tocá fierro et Don Pepe, Paris, E. Jouve, 1910.

13 Bottallo 1912 : 117-119. 
danse à Buenos Aires et à Montevideo, arrive à Paris engagé par Mademoiselle Papillon, une artiste française de variété qui aurait appris à danser le tango avec lui ${ }^{14}$.

La présence du tango à Paris apparaît ainsi en pointillé jusqu'en 1911. Dix-sept ans plus tard, Enrique Saborido expliquera, dans un entretien pour l'hebdomadaire Caras y caretas de Buenos Aires, comment il avait été invité cette année-là par Madame de Reszké à se rendre à Paris pour $\mathrm{y}$ « introduire » le tango :

J'ai accepté, et au bout de quelques mois j’obtenais mes premiers succès en France, en apprenant aux orchestres à bien jouer le tango et en donnant des leçons de danse à des personnes distinguées de la société ${ }^{15}$.

Si Saborido a bien fréquenté le salon de la comtesse de Reské, en 1911 le tango n'avait plus besoin d'être « introduit ». Le 10 janvier, Le Figaro annonçait déjà "Ce que nous danserons bientôt", à savoir «une danse argentine, le "tango argentin"». Le 20 mai, La Vie parisienne, encore indécise quant à la provenance de la nouvelle danse, indiquait que « cette saison, la mode nous viens [sic] d'Espagne : nous avons le tango ». Et l'article d'ajouter :

Qui est-ce qui n'apprend pas le tango ? Celui qui l'enseigne est la coqueluche de toutes les femmes, et l'on prétend même qu'une baronne, qui n'est pas vieille, lui accorde toutes ses faveurs - si grande est l'influence de la danse sur le cœur, ou au moins sur les sens! Dans la meilleure société, les jeunes femmes, et même les jeunes filles, s'initient aux voluptés du tango (p. 372).

Des concours de tango sont déjà organisés ${ }^{16}$, et vers la fin de l'année, Femina acte le remplacement du boston, du double ou du triple boston, qui «furent longtemps les danses à la mode dans les salons select», par le tango, qui « cette année » sera la danse « à la mode » ${ }^{17}$.

14 Fray Mocho, 28 mars 1913, p. 48 et Le Radical, 29 juin 1913, p. 4 ; Simarra remporte à Paris, avec ses partenaires la cubaine Ideal Gloria ou Maria la Bella, des concours de danse en 1912, 1913 et 1914; professeur de tango à l'hôtel Excelsior et dans l'académie de Camille de Rhynal.

15 Ernesto E. de la Fuente, «Los reyes del tango: de cómo Enrique Saborido compuso su tango "La Morocha", hace 22 años ", Caras y caretas, n¹561 du $1^{\text {er }}$ septembre 1928 ; Leiser $1934: 133$.

16 Le Figaro, 24 août 1911, p. 5 sur le succès du Bal Tabarin du 19 août.

17 «Deux nouvelles danses », Femina, ${ }^{\text {er }}$ novembre 1911, p. 582. 
Les années 1912, 1913, 1914, ne feront que confirmer et amplifier cette mode, cet engouement qui a saisi certains milieux parisiens, relayé par la presse qui rendait compte de leur goût. Le 15 juillet 1913, Femina publie sur trois pages un article signé par l'écrivain Franc-Nohain (Maurice-Étienne Legrand, 1872-1934), accompagné de huit illustrations, dont le titre aura servi à qualifier le phénomène: «Tangomanie ». Le tango, écrit Franc-Nohain, «n'est pas la première danse à laquelle la mode et le goût appliquent cette sorte de frénésie », qui « plaît toute une saison, et passe »; il a cependant ceci de particulier qu'il est "obsédant», non seulement parce que «tout le monde en parle, parce qu'on ne peut ouvrir un journal, feuilleter une revue, sans y trouver des considérations techniques, des aperçus moraux, des échos mondains, le concernant, avec l'opinion de toutes les personnalités, autorisées ou non, les plus considérables », mais aussi parce que chez les gens qui le dansent « ou qui aspirent à le danser, [...] l'obsession se manifeste singulièrement grave, et quasi morbide $»^{18}$.

Au-delà des effets de style de Franc-Nohain et d'autres qui, comme lui, s'expriment dans la même veine, les annonces et les publicités dans la presse confirment bien l'intensité singulière de la consommation du tango à Paris entre 1912 et 1914 : la prolifération des thés et des dîners-tango, des conférences, des réceptions, l'usage $\mathrm{du}$ mot pour qualifier toute sorte de produits, d'objets ou d'événements - un cocktail-tango, mélange de bière et de grenadine, une couleur-tango, allant du jaune pâle au safran en passant par l'orangé, un gâteau-tango qui était proposé dans les thés dansants, des éléments de la mode vestimentaire, un «tangomètre ${ }^{19}$, le «traintango » qui, en été, reliait Paris à Deauville, etc. Les cours de tango se multiplient, et un marché des professeurs s'installe au gré de la croissance de la demande ${ }^{20}$, toujours socialement très marquée : «La

18 Franc-Nohain $1913: 376$.

19 La Vie parisienne, 1er novembre 1913 : 1065, « petit ressort qui s'applique en manière de jarretière, au genou du danseur de tango et qui enregistre le nombre de ses pas. En principe, pour que le tangomètre donne un chiffre honorable, il faut que le danseur ait viré pendant au moins une heure ».

20 L'enseignement de la " nouvelle danse », ses lieux et ses acteurs, sont un des aspects à examiner de près pour une étude des transformations chorégraphiques du tango au cours de ces quelques années de «tangomanie » parisienne, puis européenne. 
première préoccupation des femmes élégantes [...] est d'aller apprendre chez un professeur renommé », écrit "Comtesse Éliane » dans L'Art et la mode du 3 mai 1913 ; "des châteaux environnants, on vient à Paris prendre sa leçon de "tango" " ${ }^{21}$.

Ainsi le tango «fait fureur», et «la question qui s'est posée dans les bals », dans l'hiver 1912-1913, c'est «Tanguez-vous?»22. On «tanguait» dans des résidences privées, des hôtels - le Mac Mahon Palace dans le $17 \mathrm{e}$ arrondissement, ou le Claridge, dans le $8^{\mathrm{e}}-$ des restaurants, le Palais des Glaces, les Jardins de Bagatelle, des cabarets $^{23}$, des dancings comme le Magic-City, où, d'après les Mémoires d'Elisabeth de Gramont, duchesse de Clermont-Tonnerre (1875-1954), les femmes dansaient parfois avec leur valet ou leur coiffeur $^{24}$. Comme l'écrit Georges Goursat (Sem), qui n'aimait guère cette « névrose », celle-ci avait « fait de terribles progrès » :

[...] par une marche foudroyante, elle s'est répandue sur tout Paris, a envahi les salons, les théâtres, les bars, les cabarets de nuit, les grands hôtels et les guinguettes. [...] La moitié de Paris frotte l'autre. La ville entière est entrée en branle : elle a le tango dans la peau ${ }^{25}$.

\section{Le « frottement » des corps}

Dans ce contexte, des débats se font jour entre défenseurs et détracteurs du tango, centrés principalement sur les accents excessivement sensuels, voire sexuels, de cette danse venue d'ailleurs. De fait, les interrogations sur cet ailleurs, sur les origines du tango, né dans un cadre doublement étranger - distance géographique, écart social - sont connectées aux propriétés licencieuses qu'on lui prête.

21 Comtesse Éliane 1913 : 1126-1127.

22 «La leçon de tango », L'Illustration, n³657, 20 mars 1913, p. 275.

23 «Le Loup Blanc» ou «le Princesse», rue Fontaine, le «Pigall's » rue Pigalle, «l'Apollo » ou « le Perroquet» rue de Clichy, «l'Abbaye de Thélème » ou « le Rat mort » sur la place Pigalle, «le Capitole » à Montmartre, «le Cadet Rousselle » rue Caumartin, « les Noctambules » rue Champollion, etc.

24 Gramont 1929 : 277.

25 Le texte de Sem, intitulé «Les Possédées », fut publié par tiers dans trois livraisons du Journal, le 15 avril, le 28 avril et le 18 mai 1913 [repris dans Sem 1923]. 
Le point de départ est toutefois la chorégraphie, et tout particulièrement l'une de ses caractéristiques fondamentales, à savoir le passage du couple simplement enlacé au couple étreint : le mot de Sem dans la citation précédente le dit bien, avec les connotations sans équivoque $\mathrm{du}$ verbe «frotter $»^{26}$. La marche masculine vers l'avant et l'introduction systématique d'arrêts qui interrompent le mouvement pour donner lieu aux pas ornementaux, complètent les éléments originaux introduits par le tango au sein des danses de couple. Ces trois aspects, dans l'analyse qu'en fournit Carlos Vega, sont interdépendants ; sur le plan technique, ils s'impliquent mutuellement. L'abandon du «mouvement continu »-l'enchaînement par le couple, sans s'arrêter, des pas rythmés ou des tours - plus, lorsque le couple s'immobilise soudain, l'insertion par l'homme ou la femme de figures improvisées tandis que son ou sa partenaire demeure en arrêt, rend la régularité inexistante :

[...] on ne peut rien prévoir car la figure suivante, la série complète, le thème tout entier s'élaborent à l'instant même de leur réalisation. Une technique devient nécessaire : le couple doit évoluer étreint.

Autrement dit, « les danseurs se sont posé le dilemme suivant: ou nous nous serrons l'un contre l'autre, ou nous nous marchons sur les pieds $»^{27}$.

À Paris, la disparition de la distance entre les partenaires, le fait qu'il n'y ait plus de "lumière » entre les danseurs, le contact - thoracique voire abdominal -, n'est pas perçu comme une nécessité technique, mais comme une expression de lubricité. C'est ce que dénoncent ceux qui en France - et en Europe - critiquent ou combattent l'adoption du tango, sa pratique et le goût qu'elle traduit, naif dans le meilleur des cas, plus souvent complaisant, trouble et décadent. Ainsi peut-on décrire cette «pseudo-danse qu'il faut blâmer », comme "une double danse du ventre dont la lascivité est

26 Le portail lexical du Centre national de ressources textuelles et lexicales (CNRTL) donne, parmi les acceptions du verbe «frotter », employé absolument et en argot, «Fréquenter l'autre sexe, avoir des rapports intimes avec quelqu'un» et, citant le Petit Simonin illustré dans son édition de 1968, «Transformer en jeu lascif les contacts que ménage la danse, de cavalier à cavalière » (www.cnrtl.fr/definition/frotter). 
accentuée par des contorsions exagérées $»^{28}$. Ainsi peut-on aussi, à l'instar de Sem, railler en moraliste ces « évolutions déconcertantes [qui] n'ont de la danse ni son emportement, ni son allégresse physique, ni son délire de mouvement»; pris par l'«ivresse» et le «délire» du tango, des gens "raffinés à l'excès, saturés de luxe et de confort, habitués des Ritz et des palaces», se "frottent», se "pétrissent mutuellement, avec tant d'application opiniâtre et méthodique » qu'on les croirait pratiquer du «massage abdominal». Ce n'est ni un «traitement» ni de la «culture physique», mais «un moyen de volupté », plutôt un «vice » qu'un "sport», dont on ne saurait dire si ceux qui s'y adonnent sont « des névrosés, des exhibitionnistes ou des maniaques ». Et Sem d'avouer, « devant ces contorsions mystérieuses et lascives », qu'il se « sent mal à l'aise, comme si le geste caché de l'amour était brusquement dévoilé en public $»^{29}$.

Dans cette «bataille» du tango dans le Paris des années 1912-1914, un épisode significatif eut lieu "sous la coupole», lorsque le 25 octobre 1913 l'académicien français Jean Richepin (1849-1926) prononça dans la séance publique des cinq académies de l'Institut de France, un discours «À propos du tango ». Richepin en fit l'éloge, en en évoquant une généalogie improbable où une place était accordée aux Égyptiens, mais en soulignant surtout que l'obscénité d'une danse était moins dans la danse elle-même que dans la façon de la danser ${ }^{30}$. Deux mois plus tard - le 30 décembre 1913 -, une pièce théâtrale en quatre actes, Le Tango, coécrite par Richepin et son épouse, Marie Emmanuelle de Stempowska, était créée au Théâtre de l'Athénée. Le sexe en était au cœur, l'histoire étant celle d'un jeune couple, le prince Zigi et la princesse Marie-Thérèse de Lusignan, qui n'ont pu consommer leur mariage qu'à la faveur de la danse du tango, après avoir, pour ainsi dire, tout essayé, et suivi sans succès les «fines allusions » ou les «mâles conseils» de la mère, du précepteur et du

\footnotetext{
Rivera 1913 : 9.

Sem 1913.

30 Richepin 1913. On retrouvera cet argument chez les défenseurs des danses américaines dans le Paris de l'entre-deux-guerres ; voir Jacotot 2013 : 336-342.
} 
cousin de Zigi ${ }^{31}$. Le couple en question était interprété par deux actrices, Ève Lavallière (Eugénie Fénoglio, 1866-1929) dans le rôle de Zigi, et Andrée Spinelly (Élise Fournier, 1887-1966), dans celui de la princesse de Lusignan.

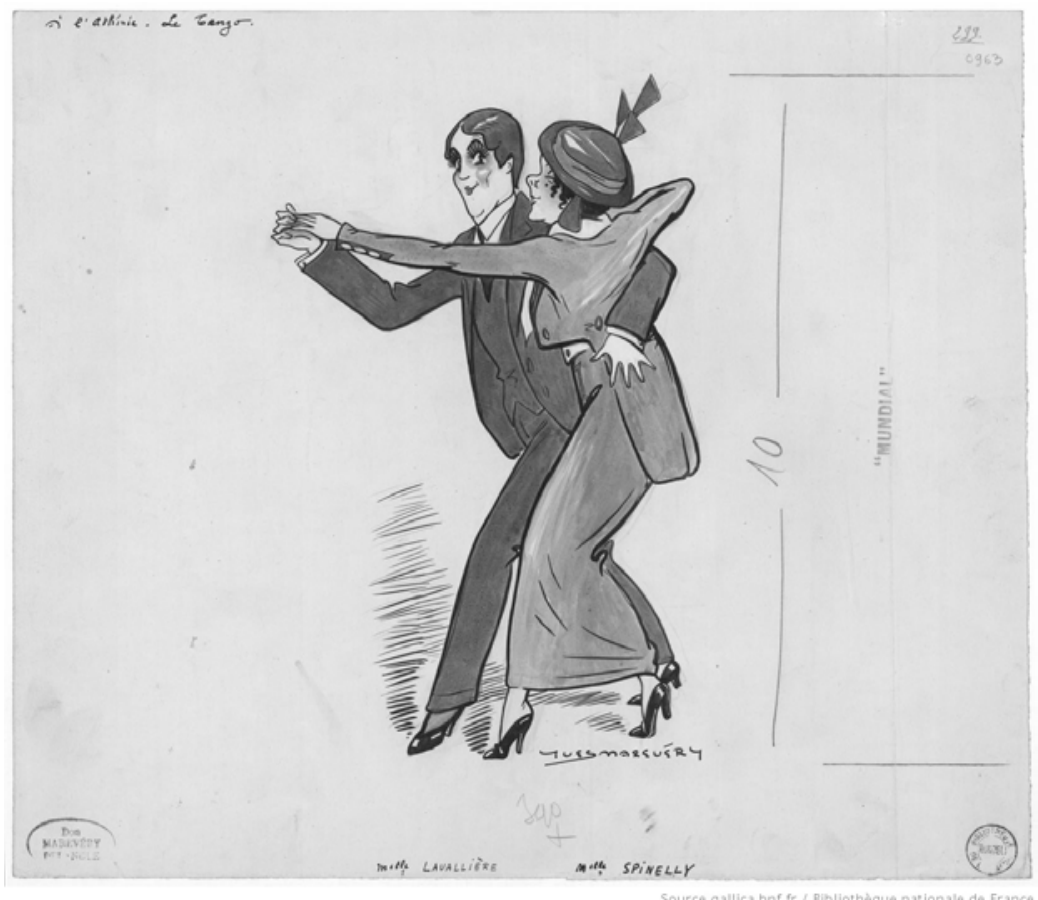

Fig. 1. Eve Lavallière et Andrée Spinelly dans «Le tango » de Jean Richepin (dessin au crayon et encre, en noir d'Yves Marevéry, 1913)

(C) BnF, Gallica.

Le tango, déjà imprégné de connotations sexuelles, venait ainsi se charger, de surcroît, d'une sorte de confusion des genres que la réception critique de la pièce ne manqua pas de souligner:

31 La pièce des Richepin est publiée en deux parties par la Vie heureuse dans ses numéros du 5 et du 20 janvier et février 1914. Gasió 1999, propose une traduction espagnole. 
Mlle de Lavallière [lit-on dans la Vie parisienne du 10 janvier 1914 (p. 33)], qui nous apparut tour à tour en jaquette, en costume de chasse, en smoking, en peignoir de bain, en pyjama, et en habit, $[\ldots]$ reculant presque les limites les plus surprenantes où le talent peut suppléer à la nature, a réussi à justifier la vigoureuse et cordiale apostrophe de son cousin, le marquis d'Orbex : «Enfin, te voilà un homme !».



Fig. 2. Mr et Mme Richepin, Spinelly et Lavallière [les deux femmes dansant le tango] (Agence photographique Rol, 1913) C BnF, Gallica.

Voilà que s’insinue le thème de la virago, cette femme masculine, aux allures, qualités et manières viriles, que Pierre Handrey évoque explicitement dans son poème "La médecine et le tango », publié dans l'Art et la mode le 27 décembre 1913 (p. 1272) :

Dansez le tango, jeunes filles!

Les jeunes filles danseront...

Et leurs formes sveltes, gentilles,

Peu à peu se transformeront.

Vous règnerez, ô viragos

Et le progrès de notre race

Sera l'ouvrage du tango. 
Voici le tango mêlé à la crainte d'une masculinisation des femmes, alors que des transformations dans l'habillement féminin vont dans le sens de permettre plus de «souplesse» aux mouvements des corps. Maintenant, écrit Flossie dans Femina, «les jupes se sont fendues, les pieds se sont dégagés, les corsets sont de simples ceintures $»^{32}$. Et Vanina, parmi beaucoup d'autres, de confirmer dans les pages de Comodia Illustré:

Si je n'ai jamais parlé ici de cette "mode du ventre" c'est que je n'ai pas douté un instant que mes abonnées n'aient compris, dès le début, qu'il s'agissait d'une attitude en pleine souplesse, d'un déhanchement gracieux, qui fait porter le haut du buste légèrement en arrière. Pour favoriser à la silhouette ces attitudes charmantes, nos corsets sont des maillots aux souples mailles, nos corsages remontent légèrement à la taille - la femme est dès lors une tige qui s'infléchit dans tous ces gestes, se couche, se penche, s'alanguit. L'influence de la danse, je ne parle pas de celle de l'ours, n'est pas pour rien dans cette esthétique nouvelle ${ }^{33}$.

D'autres craintes s'expriment qui concernent, au contraire, comme l'écrit Filippo Marinetti (1876-1944), les effets «ramollissants» du tango, dévirilisant les hommes :

$[\ldots]$ couples mollusques, félinité sauvage de la race argentine sottement amadouée, morphinisée et maquillée. Posséder une femme ce n'est pas se frotter avec mais la pénétrer ${ }^{34}$.

32 Flossie $1913: 21$.

33 Vanina 1913 : 726. Il s'agit, on s'en doute, de la «blouse-tango », « corset-tango », «culotte-tango », censés accompagner le corps des femmes dans leurs trajectoires vers davantage de « souplesse ». Chapeaux, chaussures et gants, entre autres, suivent aussi le mouvement, la taille remonte afin de bien donner de l'aisance pour faciliter les grands mouvements, on abandonne les chaînes lourdes et incommodes, de même que les sacs en forme de petites boîtes d'or ou d'argent qui pendaient parce qu'elles pouvaient frapper les jambes des danseurs.

34 Marinetti 1914, n.p. [ma traduction]. 




Fig. 4. Revue Femina, 15 mars 1914. 
Or l'homme « ramolli», la virago et les nouvelles « souplesses » des corps féminins sont des figures parmi d'autres des brouillages des rapports sexués dont le tango serait un des véhicules favorisant la manifestation publique. Écrit à la première personne, un article dans la Vie parisienne du 15 février 1913, intitulé "Un Argentin à Paris », campe celui-ci en regardant, «stupéfait» dans la nuit d'un restaurant de Montmartre, «deux grandes filles, longues, minces, souples", qui dansaient le tango «si étroitement enlacées, qu'elles semblaient ne plus former qu'un seul corps, agité des mouvements les plus tendres tantôt, et tantôt des mouvements plus expressivement sensuels ». Le désir, poursuit l'auteur, «tour à tour allumait ou adoucissait leurs yeux; leurs mains aux ongles roses et brillants, chargées de bagues, se nouaient, se desserraient, marquaient sur le dos nu et moite leur empreinte passionnée » ${ }^{35}$.

\section{L'« état civil » du tango}

Le « désir », la possibilité de se «frotter» sous prétexte de sacrifier à la mode dansante du moment, la « souplesse » des mouvements des corps que la danse du tango exige et pousse par conséquent à permettre par le biais d'évolutions de la mode vestimentaire féminine, ne sont paradoxalement que plus admis et plus suspects à la fois qu'ils reposent sur une «importation» aux origines incertaines, sinon douteuses, sujettes à caution et aptes à légitimer ou, au contraire, à condamner l'appropriation parisienne de cette danse «nonchalante et exquise des pays lointains que trop de mécréants taxent d'impudique ou de grotesque $»^{36}$.

La question de la généalogie du tango, de son contexte de création, devient un objet de discussion inséparable de celui concernant sa «décence». Ainsi Sem choisit de mettre en avant, dans sa critique, les racines du tango liées à la prostitution et aux maisons closes des capitales du Río de la Plata :

\footnotetext{
35 Fernández $1913: 120$.
}

36 Parisiana 1913b : 541. 
Laissez-nous éclater de rire en pensant que les tangos qui $[\ldots]$ portent comme titres des noms de tenancières: la Laura, la Queca, ou des propos d'alcôve de ce jus: Mordeme la Camiseta! [Mords-moi la chemise !] sont baptisés, à Paris, de petits noms doucereux et gentils, comme Loulou ou Primerose ${ }^{37}$.

Pour le journal L'Éclair, qui en juin 1913 «demande [l']état civil $»^{38}$ du tango, « le plus extraordinaire, c'est que nous ne sachions rien de cette nouvelle venue dans le monde frénétique des danses »: est-il «espagnol, argentin ou mexicain ?», se demande-t-on dans l'article, en s'en remettant à Sem, « qui le premier a dénoncé le tango comme indésirable » : il s'agirait d'un « ressouvenir du temps où les meneurs de bœuf» dansaient, "au déclin du jour, dans les énormes pampas, [...] aux accents d'une musique grave et passionnée, caractérisée par un rythme très spécial, qui rappelait un peu l'habanera et aussi les chants tristes et sauvages des Indiens aborigènes ». Or on a emprunté cette danse de "pleine nature» à ces « êtres frustes, naïfs, violents", on en a "altéré le caractère » et on l'a transportée dans les "bouges des villes", en en faisant « une danse d'une audace de gestes et d'intention inimaginable ».

$\mathrm{Si}$, une fois éduqué et francisé, le tango peut, selon Jean Richepin, devenir un passe-temps honnête - « qu'importe, en somme, l'origine étrangère et populaire d'une danse ? [...] Nous gallicisons tout »-, le directeur de la Revue Sud-Américaine à Paris, l'écrivain argentin Leopoldo Lugones (1874-1938), socialiste, puis libéral, conservateur et enfin fasciste à partir de 1924, s’insurge contre le discours de l'académicien et contre l'image peu «flatteuse pour le patriotisme argentin » qu'offrait à ses yeux le tango. Le 16 février 1914, le Mercure de France publia un long article de Lugones, qui avait assisté à la séance plénière des cinq académies et réagissait, tantôt vivement, tantôt sur un ton ironique, aux propos de Richepin ${ }^{39}$. « Nul n'ignore le caractère obscène de cette danse, écrit Lugones, quoique l'exotisme octroie volontiers un sauf-conduit à l'indécence », à la "macaquerie dernier

\footnotetext{
37 Sem 1913.

38 G.M. 1913.

39 Le Mercure de France publie, avec une introduction et un paragraphe conclusif de Lucile Dubois, un texte que Lugones avait écrit pour le journal La Nación, de Buenos Aires.
} 
cri» dont la «clientèle dansante» est composée de «sots», de «dégénérés» et de "parvenus ». Le tango est promu et pratiqué à Paris par «de petits jeunes gens sans vergogne» qui exhibent «leur nationalité de "singes des Andes" ", coupables de propager une danse qui « résume la chorégraphie du bordel ». Voici encore la prostitution, convoquée cette fois-ci par une plume censée exprimer «l'opinion des argentins eux-mêmes », qui affirme que «cette danse [...] n'est pas plus nationale que la prostitution qui l'engendra», et que les « pensionnaires des maisons closes où [le tango] naquit» ne sont pas Argentines, «sauf quelques exceptions » ${ }^{40}$. Bref, «le nom Argentin ne peut pas servir d'enseigne au bordel », s'écrie Lugones, qui prévient les «dames» : elles doivent savoir que quand elles dansent le tango « elles semblent des prostituées ». Cette «lourde pantomime », qui fait du couple « une masse si ignoble que seul le tempérament d'un nègre en peut supporter le spectacle sans répugnance ", n'a "ni grâce, ni charme, ni esthétique »; c'est une « obscénité cynique et stupide », une " "musique" africaine, sans esprit ni sentiment ${ }^{41}$.

La diatribe de Leopoldo Lugones était au fond un reproche adressé aux milieux sociaux parisiens qui avaient reçu, incorporé et promu le tango : les origines lointaines de cette forme chorégraphique avaient permis de s'en emparer au-delà des frontières sociales que sa nature aurait dû lui empêcher de franchir. L'exotisme comme alibi de la transgression, en somme, ayant pour effet, selon le folkloriste Fernand Delzangles (1871-1944), la dégradation du goût français : cette « danse pour ainsi dire professionnelle des lupanars de l'Uruguay et de l'Argentine», de même que d'autres «danses excentriques, inesthétiques, triviales», ne témoignait pas «d'un haut degré de civilisation » mais attestait plutôt «de la déchéance du goût, de la distinction et de la grâce française $»^{42}$. Or pour Lugones, l'exotisme lui-même était en réalité un malentendu, un pur produit de l'ignorance, car le tango, «ce reptile de lupanar», comme il le

\footnotetext{
40 Sur la prostitution, notamment d'origine étrangère, dans les pays du Río de la Plata entre la fin du XIXe et le début du XXe siècle, voir Trochon 2003 et 2006.

41 Dubois $1914: 871-875$.

42 Delzangles $1914: 170$.
} 
qualifiera dans un texte de $1916^{43}$, ne doit pas être pris pour «argentin»; il s'agit d'une " "musique" africaine», d'une affaire de «nègres». Africaine mais aussi italienne: ces tangos «métis et lubriques que les faubourgs italianisés de nos villes cosmopolites engendrent et propagent $[. .$.$] sous le titre de danse nationale », écrira$ encore Lugones en mai 1913, ne sont «qu'une mulâtre malhonnête engendrée par les contorsions du nègre et l'accordéon miaulant des trattorias $»^{44}$. Il y a toujours plus lubrique que soi : perversion obscène des bonnes vieilles danses des pampas sud-américaines, émanation négroïde lourdement italianisée des bouges des villes-port du Río de la Plata, le tango peut aussi être associé à ce que danserait « un couple de mahométans sous l'effet de l'opium ${ }^{45}$.

Le «monde» parisien, cependant, ne se souciait guère de ces condamnations et ces préventions ; " nous gallicisons tout », avait dit Jean Richepin, en mettant sur le compte de la francisation ce que d'autres, probablement plus avisés, situaient sur le plan social plutôt que national ou ethnique. Le chroniqueur mondain André de Fouquières (1874-1959), par exemple, n'a pas attendu Richepin pour souligner, dans une série de papiers pour le magazine Femina dès janvier 1913, que :

toute danse est susceptible d'une double interprétation: une interprétation vulgaire [...] et une interprétation distinguée, harmonieuse, conforme aux règles de la bonne compagnie [...] Les danses nouvelles [...] peuvent très bien être dansées dans le monde

et croire que «des gens corrects ne sauraient danser le tango » n'est qu'un préjugé ${ }^{46}$.

Voici le «monde» non seulement exonéré des accusations éventuelles d'inconvenance, mais identifié comme acteur d'une épuration du tango, aussi nécessaire qu'inévitable. En effet, cette danse ne peut être « importée directement. Il faut lui faire subir à la douane

\footnotetext{
43 Lugones 1916 : 174.

44 Lugones 1913, cité in Lamas, Hugo \& Binda 1998 : 131.

45 Rivera 1913 : 9.

46 Fouquières 1913a.
} 
une sérieuse visite et y apporter des modifications radicales $»^{47}$. Et Fouquières d'abonder en ce sens quelques mois plus tard :

Le tango qui se danse dans les salons est un tango de fantaisie et “aristocratisé" au goût du jour », qui « convient à l'élégance moderne ${ }^{48}$.



Fig. 3. Revue Musica, n²128, mai 1913

(Collection numérisée par l'INHA)

47 Fouquières 1913b.

48 Fouquières 1913c. 


\section{Le retour}

Dans ce même texte, un court article paru dans Le Journal du 29 novembre 1913, Fouquières s'interrogeait déjà sur la « décadence » du tango. Chaque danse, selon lui, «symbolise» une époque, c'est-àdire "une mode», et aucune autre n'avait soulevé « jamais un pareil vent de folie». En cette fin de l'année 1913, le tango « investit Paris, la province, l'Europe, et gagne le nouveau monde»; ensuite, il « reviendra comme une attraction nouvelle à Rio de Janeiro et à Buenos Ayres $»^{49}$. Sem est du même avis: quand le tango « retraversera l'Océan, vous ne le reconnaîtrez plus, belles madames de Buenos-Ayres [...]. Il vous reviendra paré de toutes les grâces de Paris, adorablement chiffonné, article de la rue de la Paix » ${ }^{50}$.

Fouquières et Sem, chacun à sa manière, ont vu juste : il y a eu un "effet de retour", une réappropriation, dans les pays Río de la Plata, du tango transformé par ce premier épisode parisien. Cette phase, à laquelle la Première Guerre mondiale mit, en Europe, un coup d'arrêt ${ }^{51}$, contribua à l'ouverture du périmètre social de consommation de cette danse à Buenos Aires et à Montevideo. L'acceptation du tango au sein de la bonne société parisienne a pu servir de caution auprès de milieux qui étaient restés rétifs ou

49 Fouquières 1913c.

50 Sem 1913.

51 Si le tango allait revenir dans l'entre-deux-guerres (voir Jacotot 2013), la presse devient quasiment muette à son égard pendant deux ans, pour n'en reparler, à partir de 1916, qu'au passé. Ainsi Maxime Weil, dans un poème publié par La Vie parisienne du 22 juillet 1916 ("En passant sur le quai d’Orsay ») (p. 553) :

Que les temps sont changés !... Sitôt que des beaux jours /

Un soleil anémique annonçait le retour, /

De « Magic » flamboyant d'ampoules électriques /

Le peuple « smart» en foule inondait les portiques... /

Et tous, dans le grand hall avec ordre introduits, /

De la Mode sur eux portant les nouveaux fruits, /

Au jeune Dieu Tango s'offraient en sacrifice... [...] C'est bien loin, le Tango...

Deux ans plus tard, dans le même périodique, un certain $\mathrm{X}$. évoquait « la dame qui en est restée à 1914. [...] elle est vieille, vieille, d'autant plus terriblement vieille qu'elle ne s'en rend pas compte. Elle date du tango dont elle n'est pas sortie, dont elle ne sortira pas » (X, La Vie parisienne du 24 août 1918, p. 739). 
hostiles à son encontre, notamment pour des raisons que l'on pourrait qualifier de socio-morales. On ne saurait réduire, cependant, l'influence de ce moment parisien à une garantie de bienséance apportée uniquement par la «marque » Paris. Il faut au moins tenir compte aussi, et peut-être principalement, des aménagements subis par la chorégraphie du tango, ayant fait l'objet d'une "sérieuse visite » à la douane et de «modifications radicales », comme l'écrit André de Fouquières. La presse en fournit de très abondantes et utiles descriptions, à l'instar de celle que donne «Parisiana » dans L'Art et la mode du 3 mai 1913 :

Dansant sur la pointe des pieds, [les danseurs] observent un silence harmonieux, les jambes pliées, procédant sans secousses, avec une heureuse tranquillité, ils donnent l'impression de gens qui se délassent, mais dont le repos aurait quelque chose du rayonnement, de l'achevé, de l'œuvre d'art. [...] D'aucuns ont reproché au tango d'être une danse qui rapetisse au lieu d'élever, mais c'est que c'est le divertissement, essentiellement moderne, de ceux qui, mélancoliques, ont abdiqué tout espoir ${ }^{52}$.

Or ces transformations de la chorégraphie, qui restent à analyser dans le détail ${ }^{53}$, ont moins été - contrairement à ce que la métaphore de la «douane» pourrait faire penser - le résultat d'une volonté explicitement engagée de rendre cette danse «honnête », que de son investissement par d'autres corps. Si une «douane » a existé, elle était surtout inscrite dans les pratiques dansantes des salons. Ainsi, une transformation de la chorégraphie qui à certains égards en a représenté un lissage, accompagna sa diffusion internationale, y compris dans ses lieux d'origine, à partir de l'épisode parisien des années 1910-1914; il en fut de même - et ce n'est peut-être pas un paradoxe - pour le développement, dans le discours, d'une forte sexualisation de l'image du tango. On danse décemment, mais on n'y pense pas moins.

52 Parisiana 1913a: 442.

53 Outre la presse, les manuels de danse sont à ce sujet une source importante, de même que des matériaux iconographiques qu'il convient toutefois de manier avec précaution. 


\section{Bibliographie}

Antología del tango rioplatense, vol. 1 (desde sus comienzos hasta 1920), 1980, Buenos Aires, Instituto nacional de musicología Carlos Vega.

Ayestarán Lauro, 1953, La Música en el Uruguay, Montevideo, SODRE.

BAtes Héctor \& Luis J. BATES, 1936, La Historia del tango, Buenos Aires, Talleres Gráficos Compañía General Fabril Financiera.

Botтallo Barthélemy G., 1912, Guide du bon danseur, Paris, Jouve \& Cie.

Bravard Alice, 2013, Le Grand monde parisien, 1900-1939. La Persistance du modèle aristocratique, Rennes, Presses universitaires de Rennes.

BuCH Esteban, 2017, «La Censure du tango par l'église de France à la veille de la Grande Guerre. Avec un post-scriptum d'Erik Satie», European Drama and Performance Studies, $8:$ «Danse et morale, une approche généalogique » (Marie GLON et Juan Ignacio VALLEjos éd.), p. 211-235.

Castro Donald S., 1990, The Argentine Tango as social history, 1880-1955. The Soul of the people, San Francisco, Mellen Research University Press.

Chasteen John Charles, 2004, National rbythms, African roots: the deep history of Latin American popular dance, Albuquerque, University of New Mexico Press.

Cocteau Jean, 1935, Portraits-somvenir, 1900-1914, Paris, Grasset.

Comtesse Éliane, 1913, «Chroniques de la vie parisienne », L'Art et la Mode, 3 mai, p. 1126-1127.

DÉCOret-AhiHa Anne, 2004, Les Danses exotiques en France, 1880-1940, Paris, Centre national de la danse.

—, 2005, "L'exotique, l'ethnique et l'authentique. Regards et discours sur les danses d'ailleurs », Civilisations, 53 : "Musiques "populaires". Catégorisations et usages sociaux » (dir. Sara LE MENESTREL), p. 149-166.

Delzangles Fernand, 1914, La Danse : son utilité... sa rénovation, Paris, J. Camber.

Dubois Lucile, 1914, «La France jugée à l'étranger. Le Tango », Mercure de France, $\mathrm{n}^{\circ} 400$, tome CVII, 25e année, 16 février, p. 871-875.

FERnÁndeZ Paul-William, 1913, « Un argentin à Paris », La Vie parisienne, 51e année, $\mathrm{n}^{\circ} 7,15$ février, p. 117-120.

FLossie 1913, «Quelques figures de danses excentriques », Femina, 1r janvier, p. 21.

FOuQuiÈRes André de, 1913a, «Les danses à la mode », Femina, 15 janvier, p. 33.

—, 1913b, «Les danses nouvelles : le tango », Femina, 1er février, p. 58.

—, 1913c, «Le Tango est-il en décadence ? ", Le Journal, 29 novembre, p. 6. 
—, 1951, Cinquante ans de panache, Paris, P. Horay.

—, 1953-1959, Mon Paris et ses Parisiens, 5 vol., Paris, P. Horay.

FranC-Nohain, 1913, «Tangomanie », Femina, n’300, 15 juillet, p. 376-378.

G.M., 1913, « Le Tango. Nous demandons son état civil », L'Éclair, 18 juin.

GASIÓ, Guillermo, 1999, Jean Richepin y el tango argentino en París en 1913, Buenos Aires, Corregidor.

Georges-Michel Michel, 1920, La Vie mondaine avant la guerre. L'époque tango, Pall Mall, Deauville, Paris, Riviera, Paris, l'Édition.

Germain José (éd.) [ca 1923], Danseront-elles ? Paris, J. Pouolozki.

GiRAUDET Eugène, 1909, Journal de la danse et du bon ton, 111-124, Paris, s.n.

—, 1914, Journal de la danse et du bon ton, 241-250, Paris, s.n.

Goldman Gustavo, 2008, Lucamba. Herencia africana en el tango, 1870-1890, Montevideo, Perro Andaluz.

Gramont Élisabeth de, 1923, Du bon ton, Paris, Ernest Flammarion.

—, 1929, Mémoires, 2 : Les marronniers en fleurs, Paris, Grasset.

—, 1966, Souvenirs du monde : de 1890 à 1940, Paris, Grasset.

Humbert Béatrice, 1995, «Le tango à Paris de 1907 à 1920 », in Ramón PeLINSKI (éd.), Tango nomade. Études sur le tango transculturel, Montréal, Tryptique, p. 109-162.

Јасотот Sophie, 2007, «Danses de société des Amériques en France dans l'entredeux-guerres », Hypothèses, 2007/1, p. 57-66.

—, 2008, "Genre et danses nouvelles en France dans l'entre-deux-guerres. Transgressions ou crise des représentations ? ", Clio. Histoire, Femmes, Sociétés, 27, p. $225-240$.

—, 2013, Danser à Paris dans l'entre-deux-guerres. Lieux, pratiques et imaginaires des danses de société des Amériques (1919-1939), Paris, Nouveau Monde éditions.

LAmAs Hugo \& Enrique BINDA, 1998, El tango en la sociedad porteña, 1880-1920, Buenos Aires, Héctor Lucci.

LeISER Clara, 1934, Jean de Reszke and the Great Days of Opera, New York, Minton, Balch \& Company.

Los Viajes de la "Sarmiento »: reseña histórica de los cruceros por todos los mares de la fragataescuela, y de la evolución de la marina de guerra argentina, Buenos Aires, Ediciones Argentinas, Raúl de Azevedo \& Cía, 1931.

Lugones Leopoldo, 1944 [1 1re éd. 1916], El Payador, Buenos Aires, Centurión. 
MarinetTi Filippo Tommaso, 1914, Abbasso il Tango et Parsifal! Lettera futurista circolare ad alcunne amiche cosmopolite che dànno dei thè-tango e si parsifalizzano, Milano, Direzione del movimento futurista.

Martin-FugIer Anne, 2003, Les Salons de la III République. Art, littérature, politique, Paris, Perrin.

Mension-Rigau Eric, 1994, Aristocrates et grands bourgeois : éducation, traditions, valeurs, Paris, Plon.

Mourgue Pierre, 1920, Eh bien, dansez maintenant, Paris, Le Bonton.

PARISIANA 1913a, «Art et chiffons », L'Art et la Mode, n¹8, 3 mai, p. 438-445.

—, 1913b, «Art et chiffons », L'Art et la Mode, n²2, 31 mai, p. 541-546.

Pringué Gabriel-Louis, 1948, 30 ans de dîners en ville, Paris, Revue Adam.

RichePIN Jean, 1913, "À propos du tango ", in Séance publique annuelle des cinq académies du... 25 octobre 1913, présidée par M. Noël Valois, Paris, Firmin-Didot.

Richepin Mme Jean \& Jean RichePIN, 1914, Le Tango, comédie en 4 actes, Supplément à La Vie heureuse, 5 et 20 janvier.

Rivera Max, 1913, Le Tango et les danses nouvelles. Tango argentin, le Double boston, la Danse de l'ours, le «One step », etc., Paris, P. Laffitte.

Rossi Vicente, 1958 [1're éd. 1926], Cosas de negros, Buenos Aires, Hachette.

Rostand Maurice, 1948, Confession d'un demi-siècle, Paris, la Jeune Parque.

Savigliano Marta, 1995, Tango and the political economy of passion, Boulder, Westview Press.

SEM 1913, «Les Possédées », Le Journal, 15 avril, p. 1 ; 28 avril, p. 1 ; 18 mai, p. 1.

—, 1913b, Tangoville sur mer, s.l., Succès.

—, 1923, La Ronde de nuit, 120 dessins de Sem ; gravure de L. André, Paris, Fayard.

SUQueT Annie, 2012, "Exotisme(s) et modernité(s) : l'Autre en miroir », in L'Éveil des modernités. Une histoire culturelle de la danse (1870-1945), Paris, Centre national de la danse, p. 255-313.

Tango-Rausch. Künstler Album mit 20 Meisterbildern, 1913, Berlin, Lustige Blätter.

Trochon Yvette, 2003, Las Mercenarias del amor. Prostitución y modernidad en el Uruguay (1880-1932), Montevideo, Taurus.

-, 2006, Las Rutas de Eros. La Trata de blancas en el Atlántico sur (Argentina, Brasil, Uruguay) (1880-1932), Montevideo, Taurus.

VANINA, 1913, «La Comédie de la Mode », Comæedia Illustré, 5 mai, p. 717-728.

VEGA Carlos, 1936, Danzas y canciones argentinas: teorías e investigaciones. Un ensayo sobre el tango, Buenos Aires, Eugenio Ferrero. 
—, 1952, Las Danzas populares argentinas, Buenos Aires, Ministerio de Educación de la Nación, Instituto de Musicología.

—, 1967, «Las especies homónimas y afines de "Los orígenes del tango argentino" », Revista Musical Chilena, 101, p. 49-65.

VILARIÑo Idea, 1964, «Los tres lenguajes del tango », Número, 2/3-4, p. 23-42.

—, 1965, Las Letras de tango: la forma, temas y motivos, Buenos Aires, Shapire.

—, 1981, El Tango cantado, Montevideo, Calicanto.

WarnoD André, 1922, Les Bals de Paris, Paris, G. Grès \& Cie.

WeIl Maxime, 1916, «En passant sur le quai d'Orsay», La Vie parisienne, 22 juillet, p. 553.

X., 1918, «La dame de 1914 », La Vie parisienne, 24 août, p. 739-740. 